\title{
Desarrollo de un Modelo 3D para un Sistema Infotainment
}

\author{
Development of a 3D Model for an Infotainment System
}

\author{
Andreé M. Nava-García ${ }^{1}$ (D), Huizilopoztli Luna-García ${ }^{1}$ (D), José Ma. Celaya-Padilla ${ }^{1}$ (D),

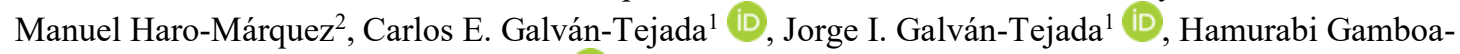 \\ Rosales $^{1}$ (iD), Luis Alberto Flores-Chaires ${ }^{1}$ \\ ${ }^{1}$ Universidad Autónoma de Zacatecas, Zacatecas, Zac. México \\ ${ }^{2}$ Consejo Zacatecano de Ciencia, Tecnología e Innovación (COZCyT), Zacatecas, Zac. México \\ navandree00@gmail.com, \{hlugar, jose.celaya, ericgalvan, gatejo, hamurabigr, luischaires\}@uaz.edu.mx, \\ mharo@cozcyt.gob.mx
}

(Recibido: 21 Septiembre 2020; aceptado: 22 Octubre 2020; Publicado en Internet: 1 Diciembre 2020)

\begin{abstract}
Resumen. En la actualidad somos testigos del constante avance en el desarrollo de tecnologías para la industria automotriz donde cada día hay avances y mejoras significativas para brindar mayor comodidad a los pasajeros. Una de las tecnologías del vehículo que está especialmente en desarrollo es el diseño de interfaces de usuario específicas para sistemas infotainment y diferentes tipos de interacción, por ejemplo, la implementación de interfaces de usuario 3D para proporcionar retroalimentación al usuario. Estas interfaces se pueden crear a partir de tecnologías disponibles en el mercado, tanto de software libre como privado para el diseño de modelos 3D y para el desarrollo de Interfaces Humano Máquina utilizados en la industria automotriz. Este artículo describe el proceso que se llevó a cabo para el desarrollo de una interfaz funcional a partir de tecnologías para el modelado 3D y diseño de interfaces. Dicha interfaz proporciona una retroalimentación visual al usuario que permite comprender lo que sucede en el vehículo.
\end{abstract}

Palabras clave: Interacción Humano Máquina (HMI), Interfaces de Usuario Automotrices (AUI), Sistemas Infotainment, Modelos 3D, Industria Automotriz.

\begin{abstract}
At present, we are witnessing the constant advance in the development of technologies for the automotive industry. Every day, there are significant advances and improvements to provide greater comfort to passengers. One of the vehicle technologies that are especially under development is designing specific user interfaces for infotainment systems and different types of interaction, for example, the implementation of 3D user interfaces to provide feedback to the user. These interfaces can be created from technologies available on the market, both free and private software for designing 3D models, and the development of Human Machine Interfaces used in the automotive industry. This article describes the process followed to develop a functional interface from technologies for 3D modeling and interface design. This interface provides visual feedback to the user that allows understanding of what is happening in the vehicle.
\end{abstract}

Keywords: Human Machine Interface (HMI), Automotive User Interfaces (AUI), In-Vehicle Infotainment (IVI), 3D models, Automotive Industry.

Tipo de artículo: Artículo de investigación.

\section{Introducción}

A lo largo de los años la sociedad ha generado grandes avances tecnológicos, los cuales al ser de amplio espectro y ser trascendentales por su potencial para transformar las vidas humanas, han permitido crecer, resolver problemas, cubrir necesidades, y sobre todo facilitar las actividades desarrolladas en la vida cotidiana (West, 2016). En este sentido la tecnología actualmente juega un papel muy importante en la vida cotidiana de los seres humanos.

Las nuevas tecnologías se han aplicado en diferentes áreas del conocimiento y/o la industria, por ejemplo, la educación, seguridad, vivienda y medios de transporte. Específicamente la industria automotriz, ha desarrollado e implementado nuevas tecnologías que permiten que el conductor y pasajeros disfruten de una mejor experiencia de usuario en términos de comodidad, conducción, entretenimiento y seguridad, por mencionar algunos. En cuanto a la conducción se han incorporado diferentes sistemas, por ejemplo, sistema de transmisión y frenado; suspensión y dirección; mientras que la cabina del auto cuenta con sistemas de 
infoentretenimiento, mejor conocido en la industria automotriz como Sistema Infotainment. De acuerdo con Saxena (2018), un sistema infotainment combina la entrega de información del vehículo con el entretenimiento, además busca facilitar la interpretación de información al conductor y pasajeros.

En este sentido, Luna-García et al., (2017) menciona que los sistemas IVI (In-Vehicle Infotainment) han creado un nuevo entorno de comunicación entre el conductor, pasajeros y automóvil, permitiendo el desarrollo de aplicaciones con un amplio abanico de posibilidades de interacción, comunicación y colaboración entre conductores, pasajeros y el entorno. Al respecto, Althoff et al., (2003) refieren que un enfoque de diseño en 3D para sistemas infotainment, podría aumentar la usabilidad general al interactuar con sistemas de información complejos en el automóvil mediante la aplicación de patrones de visualización agradables.

Es importante mencionar en este punto que, el desarrollo que se muestra en este artículo, forma parte de un proyecto específico en el Centro de Investigación e Innovación Automotriz de México (CIIAM), a través del cual se pretende contar con el desarrollo tecnológico propio de un Sistema Infotainment con la funcionalidad completa disponible en Sistemas Infotainment similares en el mercado. El propósito de este proyecto es desarrollar tecnología útil, fácil de usar y segura para conductores y pasajeros. De la misma manera, una de las metas del proyecto es implementar Software Libre y Hardware de bajo costo, con la finalidad de hacerlo asequible a la población que no puede adquirir un vehículo de gama media-alta, que es donde normalmente se instala un Sistema Infotainment. De acuerdo con lo anterior, en este artículo, se describe el proceso que se realizó para el diseño de un modelo en 3D utilizando Blender y posteriormente su integración en la plataforma EB Guide de Elektrobit ${ }^{1}$. A continuación, se describen brevemente algunas plataformas para el desarrollo de Sistemas Infotainment y Modelado en 3D con el propósito de contextualizar la tecnología disponible en el mercado.

\subsection{Plataformas HMI para el diseño de interfaces en sistemas infotainment}

Un sistema infotainment (IVI), es una Interfaz Humano Máquina (IHM, por sus siglas en inglés) que permite la comunicación entre el usuario con el sistema del automóvil mediante una interfaz gráfica (Hix \& Hartson, 1993). Un sistema IVI utiliza interfaces de audio y video, pantallas táctiles, entre otros dispositivos para funcionar y proporcionar diferentes servicios.

A continuación, se muestran algunos softwares para desarrollar Sistemas Infotainment Automotrices.

- Automotive Grade Linux (AGL), es un proyecto colaborativo de código abierto que reúne a fabricantes de automóviles, proveedores y empresas de tecnología para acelerar la adopción de una pila de software completamente abierta para el automóvil conectado (Automotive Grade Linux, 2020).

- Automotive Grade Android (AGA), ofrece un sistema operativo de código abierto, para que fabricantes de dispositivos integren aplicaciones Android en un sistema IVI. AGA ha desarrollado un conjunto de principios de diseño para el uso seguro de aplicaciones tomando de base los principios ya existentes de Android para el desarrollo de aplicaciones móviles (Automotive Grade Android, 2020).

- HARMAN, diseña productos y soluciones conectados para fabricantes de automóviles, consumidores y empresas de todo el mundo, incluidos sistemas de automóviles conectados, productos de audio y visuales, soluciones de automatización empresarial (Harman, 2020).

- EB Guide Studio, pertenece a la empresa Elektrobit. Es una herramienta versátil para el desarrollo inteligente de productos IHM multimodales. El software de EB Guide Studio ofrece soluciones flexibles e innovadoras para software de infraestructura para automóviles, conectividad, seguridad, conducción automatizada, y herramientas relacionadas (Elektrobit, 2020).

El Centro de Investigación e Innovación Automotriz de México (CIIAM) perteneciente a la Universidad Autónoma de Zacatecas (UAZ) ha desarrollado prototipos funcionales utilizando la plataforma "EB Guide Studio", con la intención de generar tecnología propia. El entorno de EB Guide permite la creación de Interfaces Humano Máquina (IHM) para unidades automotrices, clusters infotainment y sistemas de navegación.

\footnotetext{
${ }^{1}$ El Centro de Investigación e Innovación Automotriz de México (CIIAM) cuenta con el convenio "University Program" de la empresa Elektrobit para el uso de la plataforma de diseño EB Guide Studio.
} 


\subsection{Modelado en 3D}

La interacción en tres dimensiones tiene un sentido intuitivo para una amplia gama de aplicaciones debido a las características de las tareas en estos dominios y su coincidencia con las características de los entornos 3D (Jr LaViola et al., 2017), por ejemplo, los entornos gráficos proporcionan mayor entendimiento de lo que sucede, es por ello que tiene mejor tiempo de respuesta lo que favorece la relación entre el usuario y el sistema.

Para una mejor experiencia de usuario es necesario definir los requerimientos del modelo, como las características que debe cumplir acorde al objetivo. Derivado de lo anterior existen diferentes softwares para el diseño de Modelos en 3D en el mercado que permiten cumplir las características necesarias del modelo a desarrollar. Algunos softwares son parte de la comunidad de Software Libre u Open Source, por ejemplo, OpenSCAD, SketchUp, Blender, Sculptris, SculptGL, entre otras. Asimismo, existen plataformas de software privado o licenciado como, 3D Splash, AutoCAD, Autodesk Maya, Clara.io, ZBrush entre otras. A continuación, se describen las características más relevantes de algunas de estas plataformas que fueron comparadas para cumplir con el propósito de este proyecto de desarrollo, que es generar un modelo 3D para una interfaz de sistema infotainment automotriz.

- ZBrush, es un programa de modelado que tiene una capacidad de redefinir localmente la estructura interna del modelo. Este software tiene un enfoque principal en el concepto de plastilina o arcilla por lo que cuenta con un conjunto de pinceles que emulan los efectos de las herramientas de escultura del mundo real, además se puede pintar texturas de manera directa en el modelo (Pixologic, 2020).

- 3D Slash, plataforma de software licenciado (aunque tiene una versión libre), ofrece una original e intuitiva experiencia de modelado en 3D lo que lo hace muy sencillo de manipular. Se modela alrededor de bloques de construcción. Los bloques se pueden eliminar y combinar utilizando diversas herramientas (3DSlash, 2020).

- Blender, es un proyecto público de código abierto para el modelado 3D, que admite la totalidad de la canalización 3D: modelado, aparejo, animación, renderizado, composición y seguimiento de movimiento. También es una excelente herramienta para la producción de efectos visuales. Cuenta con un API en Python, y esta puede ser personalizada de acuerdo con los requisitos de cada proyecto (Blender, 2020).

- Clara.io, es una herramienta de modelado 3D, animación y renderizado basada en la nube con todas las funciones que se ejecutan en el navegador. Clara.io cuenta con herramientas de modelado como: extrusión, biselado, corte y bucle. Esto supera las expectativas de un programa de modelado en el navegador, por lo que permite crear modelos complejos sin instalar ningún otro programa (Clara.io, 2020).

Es posible apreciar que existen diferentes alternativas disponibles en el mercado para los diseñadores y desarrolladores de Modelos 3D.

Finalmente se seleccionó como entorno de diseño la plataforma Blender, principalmente por ser de código abierto y porque brinda una amplia variedad de herramientas para el modelado. Como plataforma de desarrollo para la Interfaz del Sistema Infotainment y la integración del Modelo en 3D, se utilizó EB Guide Studio derivado del convenio que se tiene con la empresa Elektrobit. Sin embargo, como se mencionó anteriormente una de las metas es utilizar Software Libre. El modelo en 3D que se desarrolló es un automóvil que permite una funcionalidad segmentada en puertas, cofre y cajuela. Estas características fueron determinadas tomando de base las necesidades y objetivos iniciales del proyecto, las cuales se describen brevemente a continuación:

- Funcionalidad segmentada: puertas, cofre y cajuela deben ser objetos independientes, de esta manera cada objeto tendrá su centro de rotación, esto servirá para manipular mediante código las funciones de cada objeto.

- Geometría: el modelo debe de mantenerse en cuadriláteros, de esta manera al añadir modificadores la pieza modelada no se distorsiona.

- Visualización: Se debe integrar una cámara e iluminación adecuada con el propósito de proporcionar una visualización correcta durante la manipulación del modelo $3 \mathrm{D}$ en la plataforma EB Guide Studio. 
Finalmente, el modelo es manipulado mediante botones en la pantalla de un Smartphone que activan las funciones de apertura y cierre del Modelo en 3D, de esta manera se da una retroalimentación visual al usuario.

\section{Proceso de desarrollo}

El proceso que se implementó para el diseño y desarrollo del Modelo 3D, su integración en EB Guide Studio y despliegue en un Smartphone, se muestra en la Figura 1.

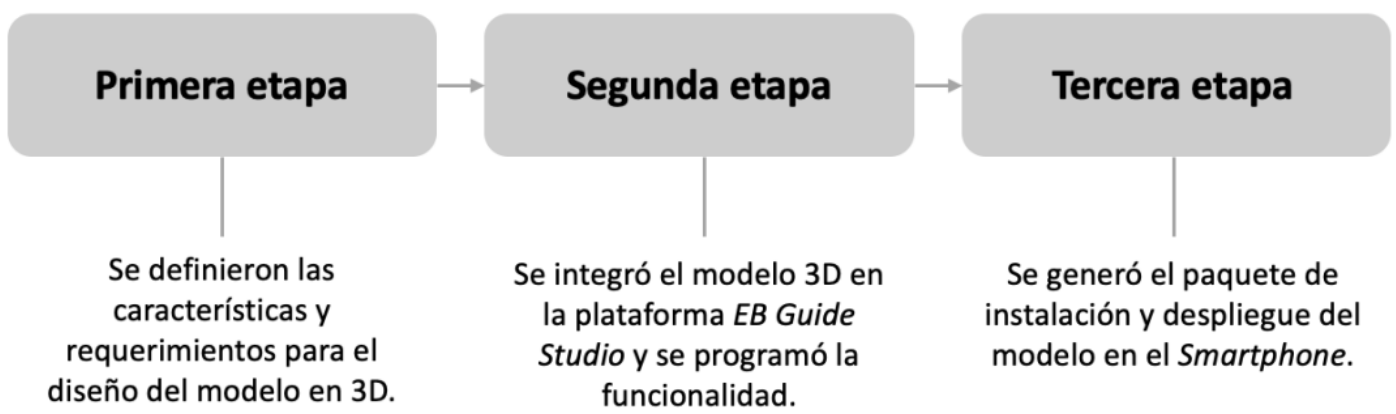

Figura 1. Proceso de desarrollo implementado.

\section{Diseño del Modelo en 3D}

Como se mencionó en la sección 1.2, Blender se utilizó como plataforma de diseño para el modelo 3D con la intención de que este fuera "low poly", es decir, de malla poligonal, la cual contempla un número pequeño de líneas, con el propósito de mantener la geometría del modelo y optimizar el rendimiento. Para el modelado se utilizó un blueprint ${ }^{2}$ y un plano como referencia para el diseño del modelo (ver Figura 2).

\footnotetext{
${ }^{2}$ Blueprint, plano de diseño donde están las diferentes vistas de un objeto.
} 


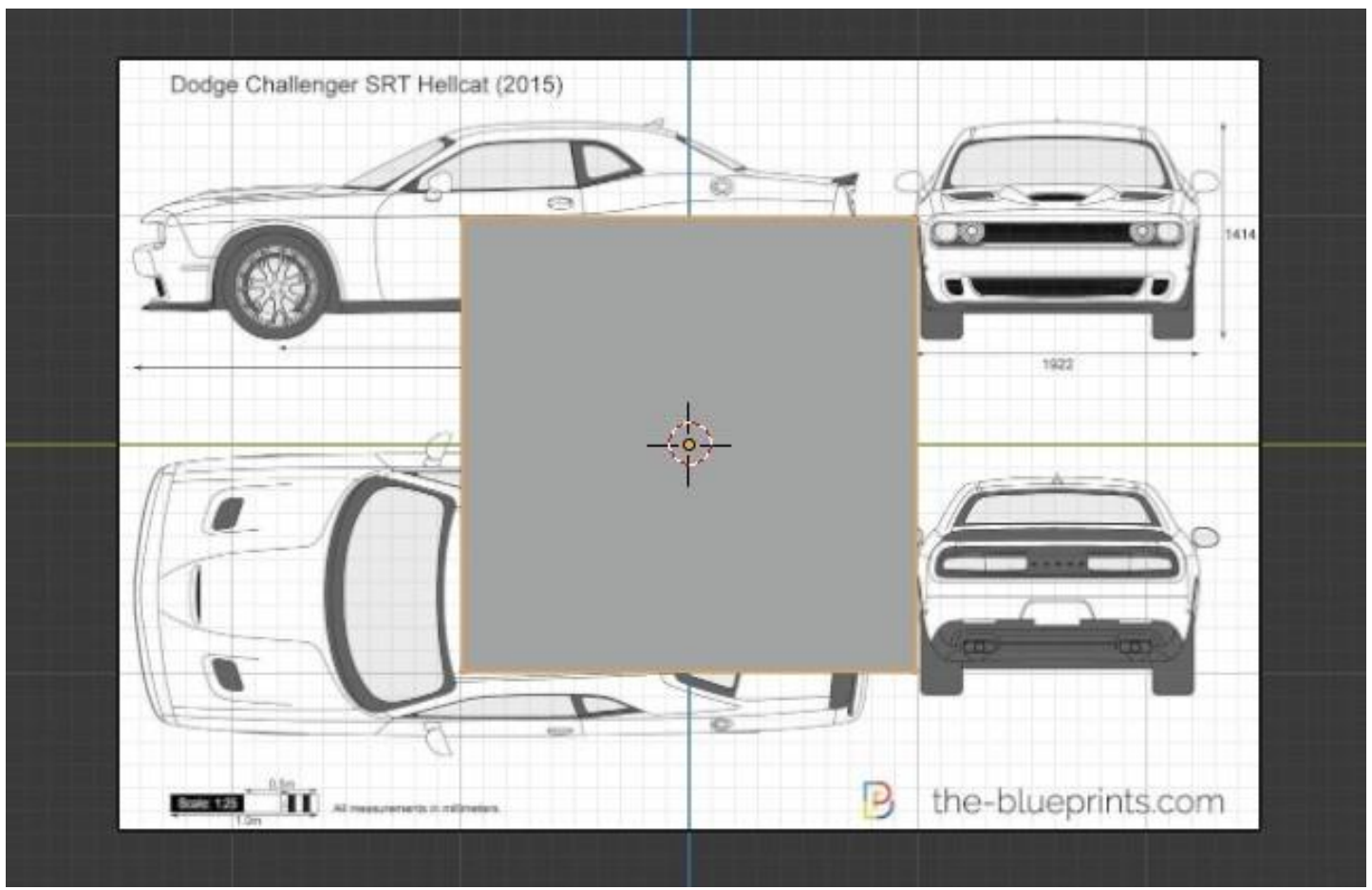

Figura 2. Blueprint utilizado y el plano gris para modelar

El plano gris mostrado en la Figura 2 fue ajustado al tamaño necesario para modelar el techo del vehículo. Con ayuda de la vista superior, fue posible reducir y ajustar las medidas necesarias para darle forma, asimismo, sucedió con las vistas laterales para dar línea y forma a la figura del automóvil (ver Figura 3).

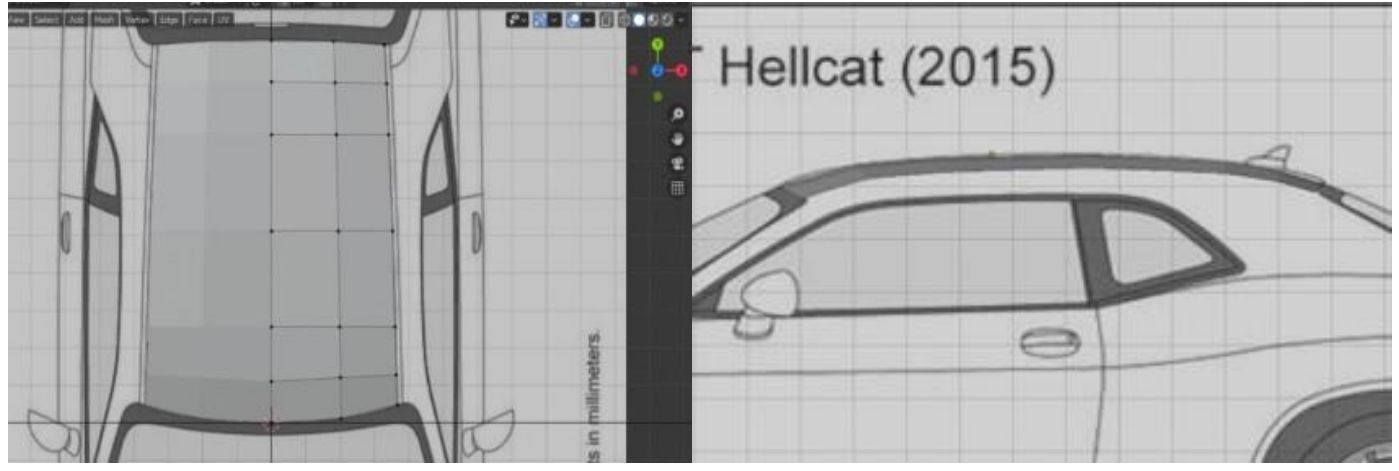

Figura 3. Vista superior y lateral del plano

Cuando se diseña un modelo tomando de base un blueprint, es necesario manipular las vistas para diseñar la forma correcta, de esta manera se modelaron las piezas del vehículo (Modelo 3D) omitiendo las piezas móviles tales como puertas, cofre y cajuela. Como se mencionó anteriormente, estos objetos deben ser partes independientes al resto del vehículo con el propósito de dotarlas de funcionalidad. Al ser piezas independientes cada una tiene su centro de rotación lo que permite ejecutar acciones y dotarlas de funcionalidad, para este caso, abrir o cerrar (ver Figura 4). 

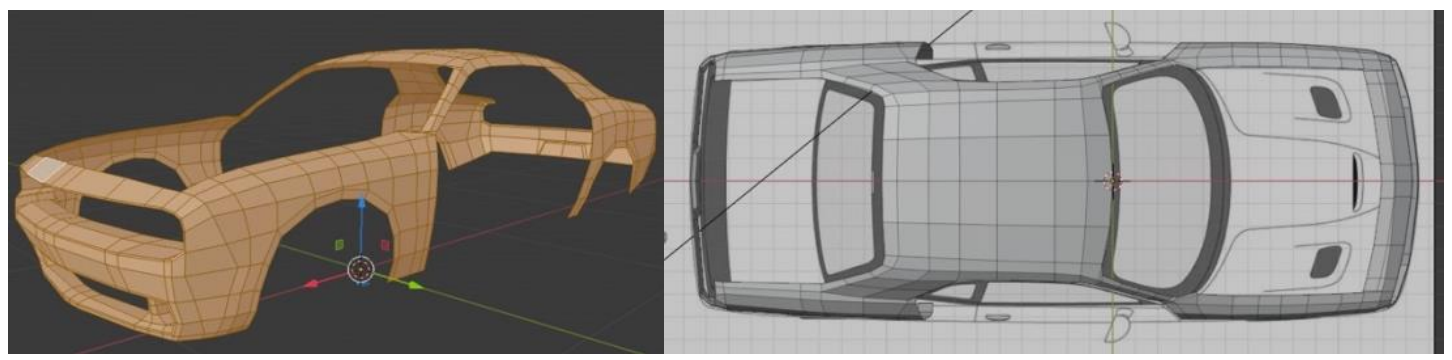

Figura 4. Modelo sin las piezas que tendrán funcionalidad (Vista lateral y superior)

Para el diseño de las piezas independientes (cofre, puertas y cajuela), el procedimiento fue similar ya que se tomó un plano y de la misma manera se manipularon las vistas para dar la forma adecuada y seguir con las líneas del vehículo. La diferencia es que estas piezas tienen el centro de rotación en el lugar donde se ubican las bisagras en un vehículo real (ver Figura 5).

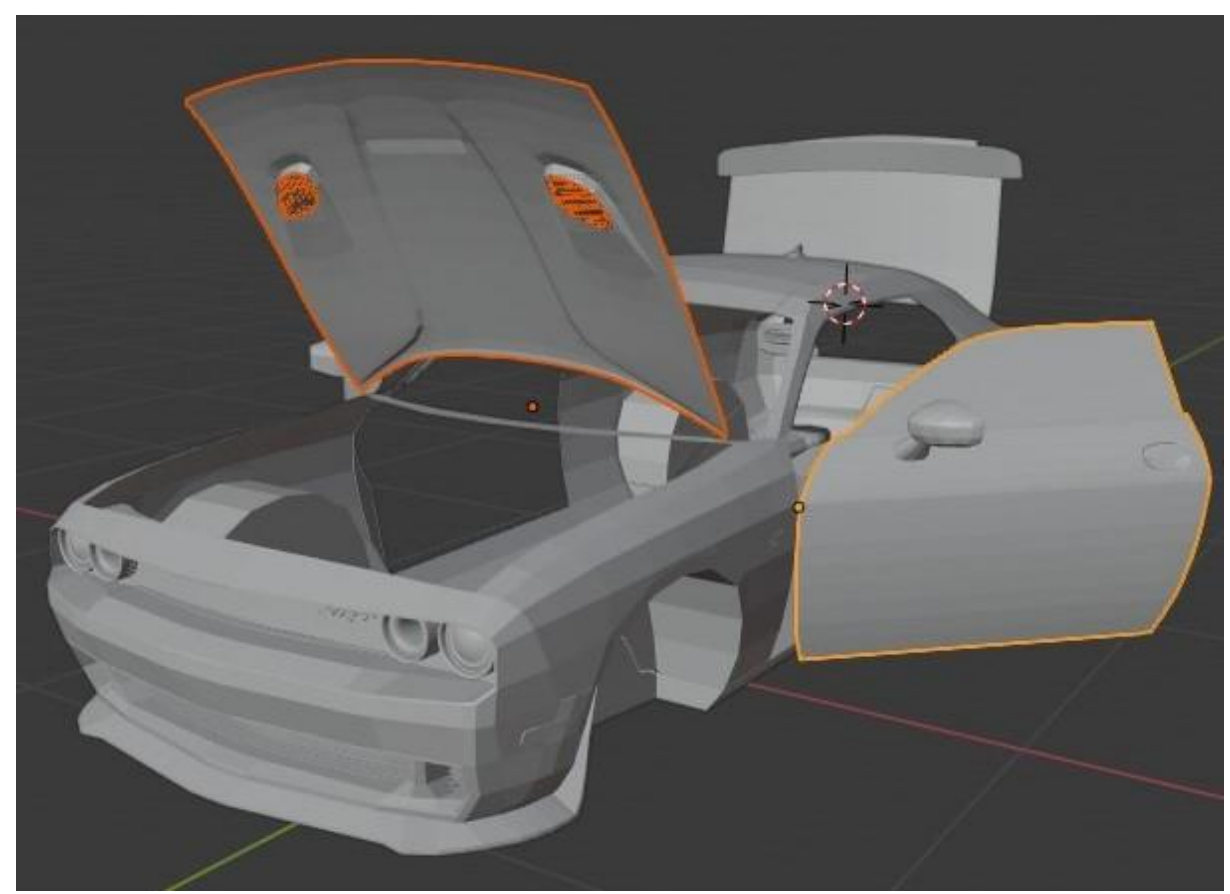

Figura 5. Piezas independientes donde los puntos amarillos indican los centros de rotación

En la Figura 6 se muestra el Modelo 3D Low poly (izquierda) y con texturas del vehículo diseñado (derecha).

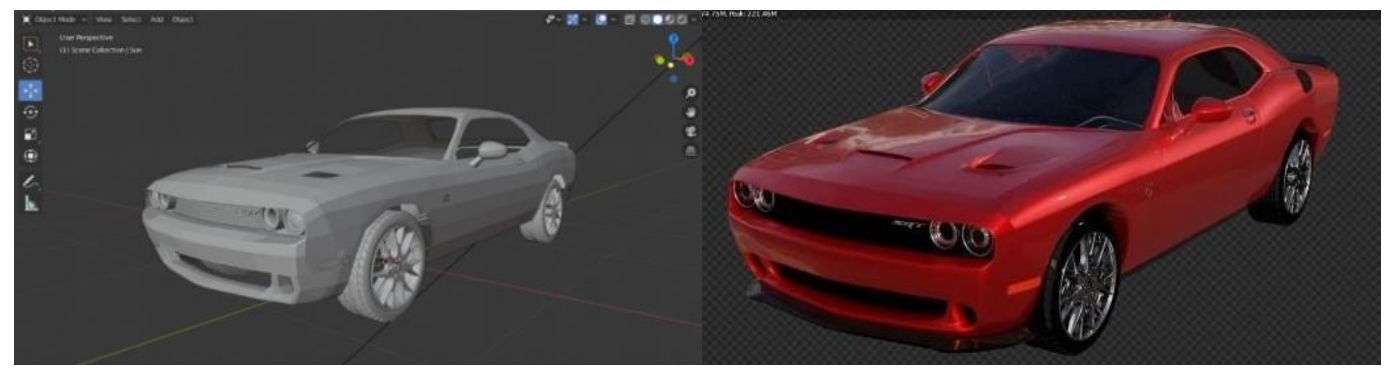

Figura 6. Modelo low poly terminado (izquierda) / Modelo con texturas (derecha) 


\section{Integración del Modelo en la plataforma EB GUIDE}

La siguiente fase dentro del proceso, corresponde a la integración del Modelo 3D en la plataforma EB Guide Studio. Este IDE implementa el modelo de "Máquinas de Estado". Cada máquina de estado es un dispositivo que almacena el estado de un sistema en un tiempo determinado. Dicho estado cambia de acuerdo con las entradas, las cuales sirven como parámetros para implementar cambios y producir una salida o resultado, asegurando que siempre se estén ejecutando las acciones correspondientes al estado actual, y evaluando si se cumple alguna condición de las transiciones que parten de dicho estado (LunaGarcia et al., 2017), de esta manera es posible mostrar una retroalimentación visual al conductor o pasajeros sobre la funcionalidad programada en el Modelo 3D. La Figura 7 muestra una captura del proceso de diseño de la interfaz IHM-IVI usando las herramientas proporcionadas por la plataforma.

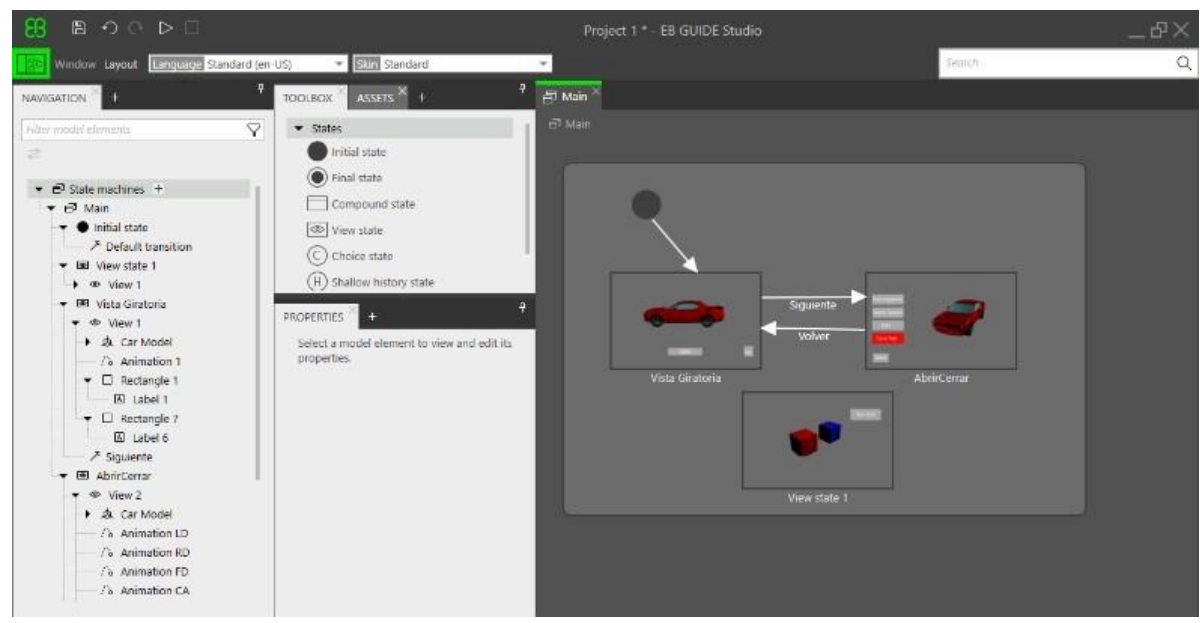

Figura 7. Diseño de la interfaz usando EB Guide Studio ${ }^{\circledR}$

Como puede observarse en la Figura 7, en la interfaz se utilizan dos "View state", las cuales contienen el Modelo 3D diseñado y, la Figura 8 muestra los botones que permiten activar la funcionalidad del modelo, es decir, girar el vehículo (izquierda) o abrir y cerrar las puertas, cajuela y cofre del vehículo (derecha).

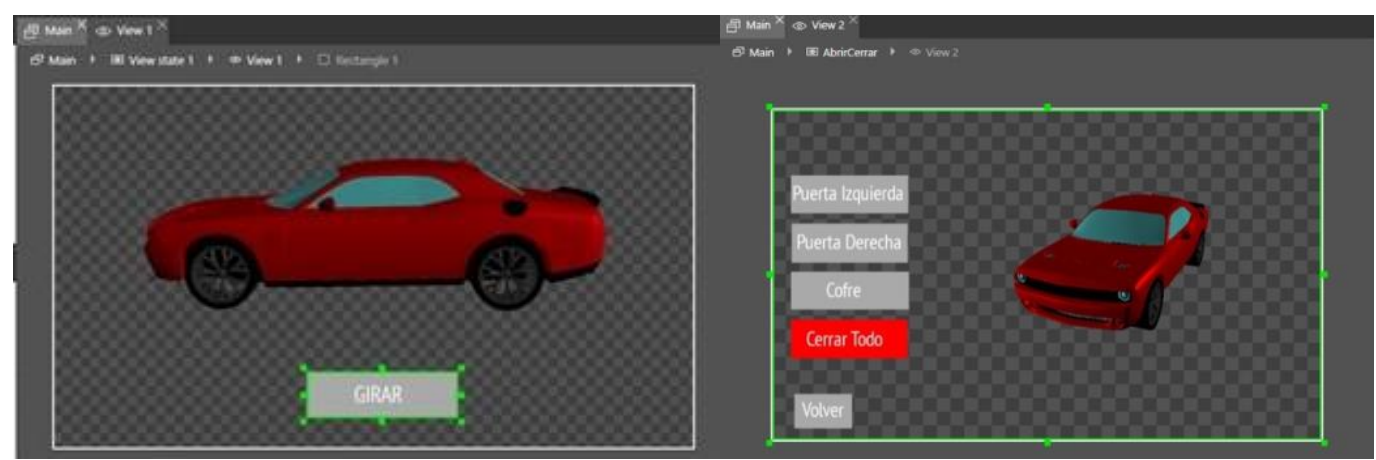

Figura 8. Botones que permiten manipular la funcionalidad del Modelo 3D en EB Guide Studio

Para la programación de la funcionalidad de las piezas (movimientos) se realizó una interpolación lineal y una rotación en el eje correspondiente a la dirección donde se rotará, esto con el propósito de que el cofre, puertas y cajuela del modelo se abran o cierren dependiendo del evento que esté activado. En la Figura 9 se muestra el modelo con todas las piezas móviles abiertas (izquierda) y el programa correspondiente para ejecutar la animación (derecha). 


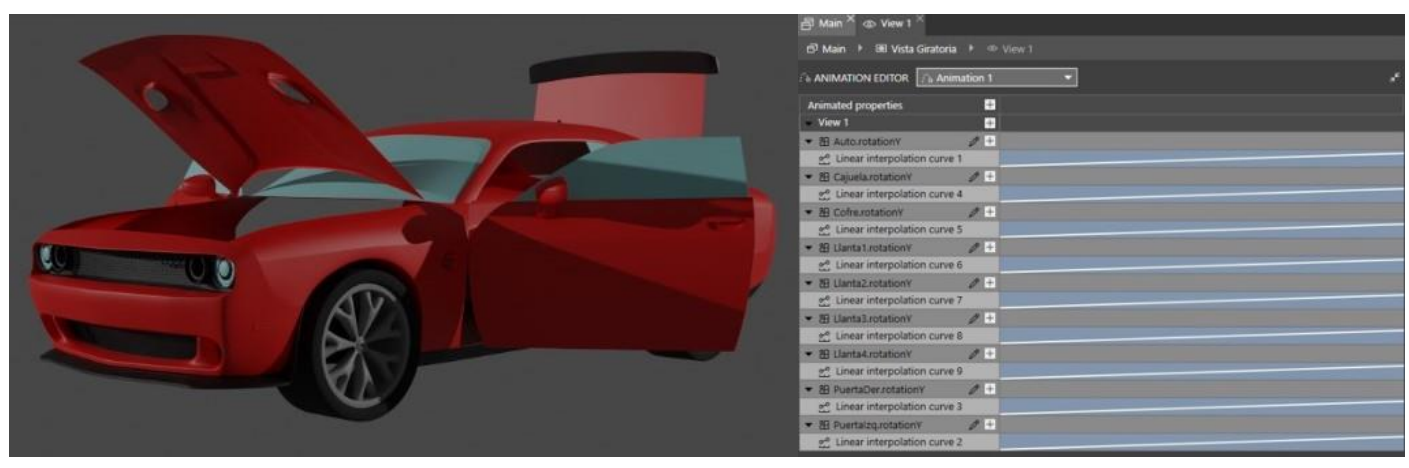

Figura 9. Partes móviles del Modelo 3D (izquierda), secuencia de instrucciones para la animación (derecha)

En este punto del proyecto, es necesario activar la funcionalidad de las piezas móviles a través de botones ubicados en la misma interfaz y visualizar la simulación en la pantalla. Como se mencionó en la Sección 1 este trabajo forma parte de un proyecto en proceso que busca finalmente integrar este sistema IVI en un vehículo real donde los sensores y/o actuadores ubicados en las puertas, cajuela y cofre del vehículo activen la funcionalidad del Modelo 3D y se visualice en la pantalla ubicada en el vehículo.

\section{Despliegue}

Una vez desarrollada la interfaz esta puede ser exportada y desplegada en diferentes plataformas, entre ellas, los smartphones con Sistema Operativo Android o la plataforma Raspberry Pi. En la Figura 10 se muestra la implementación del sistema IVI funcionando en un Smartphone.

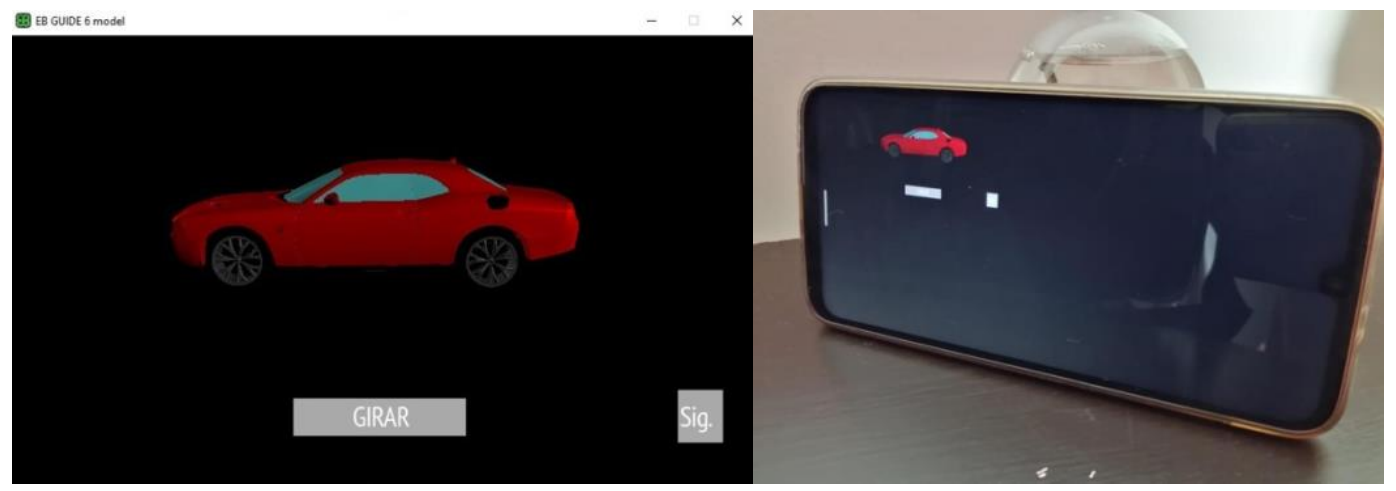

Figura 10. Implementación de la IHM en un Smartphone

Es importante mencionar que la interfaz se diseñó con la resolución propia para una pantalla típica de Sistemas Infotainment (800 x 480). Para la prueba de despliegue inicial se seleccionó un smartphone con una resolución mayor, motivo por el cual, la interfaz se visualiza más pequeña, sin embargo, esta primera prueba se enfocó en probar la flexibilidad de la plataforma para el despliegue de Modelos IHM-IVI 3D en diversas plataformas, en este caso, un smartphone.

\section{Conclusiones}

En este artículo se describen algunas de las tecnologías disponibles en el mercado para el diseño de Modelos 3D, asimismo, diversas plataformas disponibles para la integración de Modelos 3D y desarrollo de interfaces para Sistemas Infotainment para la industria Automotriz. Se realizó un análisis que permitió comparar y vincular plataformas de Diseño de Modelos 3D e Integración, Desarrollo y Despliegue de Interfaces IHM para la Industria Automotriz (Sistemas Infotainment con Modelos en 3D). Se seleccionó 
Blender como tecnología para el Desarrollo de un Modelo de vehículo en 3D y EB Guide Studio como plataforma de Integración, Desarrollo y Despliegue. Como resultado se desarrolló un prototipo de interfaz de Sistema Infotainment con Modelo en 3D para la industria Automotriz.

Como trabajo futuro, se pretende migrar la interfaz IHM-IVI desarrollada a una plataforma basada en Raspberry Pi, y generar un primer prototipo con sensores y/o actuadores que activen la funciónretroalimentación de la interfaz en una pantalla HMI. Asimismo, mejorar el prototipo presentado en este artículo y posteriormente realizar pruebas de funcionalidad y experiencia del conductor.

\section{Agradecimientos}

Los autores agradecen al Laboratorio de Software Libre (LABSOL) del Consejo Zacatecano de Ciencia y Tecnología (COZCyT) por el apoyo otorgado bajo el programa de Becas LABSOL durante el periodo Enero - Mayo 2020. Asimismo, el autor principal, agradece al CIIAM por el apoyo durante la realización de este proyecto de desarrollo tecnológico.

\section{Declaración de conflicto de intereses}

Los autores declaran no tener conflicto de intereses con respecto a la investigación, autoría o publicación de este artículo.

\section{Referencias}

3DSlash. (2020). 3D Slash - a 3D piece of cake. https://www.3dslash.net/news about.php

Althoff, F., McGlaun, G., Lang, M., \& Rigoll, G. (2003). Comparing an Innovative 3D and a Standard 2D User Interface for Automotive Infotainment Applications. En G. Szwillus \& J. Ziegler (Eds.), Mensch \& Computer 2003 (pp. 53-63). Vieweg+Teubner Verlag. https://doi.org/10.1007/978-3-322-80058-9 7

Automotive Grade Android. (2020). Develop an Android Device. https://source.android.com/devices

Automotive Grade Linux. (2020). Automotive Grade Linux (AGL). https://www.automotivelinux.org/

Blender. (2020). Blender. https://www.blender.org/

Clara.io. (2020). Clara.io, Online 3D modeling. https://clara.io

Elektrobit. (2020). EB Elektrobit. https://www.elektrobit.com/

Harman. (2020). Harman Samsung Company. HARMAN International. https://www.harman.com/

Hix, D., \& Hartson, H. R. (1993). Developing user interfaces: ensuring usability through product and process. Wiley.

Jr LaViola, J. J., Kruijff, E., McMahan, R. P., Bowman, D., \& Poupyrev, I. P. (2017). 3 D User Interfaces: Theory and Practice (2nd Edition) (T. Mark, L. Lewin, S. Zahn, S. Schroeder, L. Lyons, \& W. Hapke (eds.)). AddisonWesley.

Luna-Garcia, H., Arceo, J. G., Gamboa Rosales, H., Celaya Padilla, J. M., Galván Tejada, C. E., Monteagudo López, F., González, A., Moreno Baez, A., \& Tejada Galván, J. I. (2017). Diseño de Interfaces Infotainment de Bajo Costo para la Industria Automotriz. En 18th Edition of the International Conference Interacción 2017 (Ed.), Congreso Internacional Interacción 2017 (pp. 25-27).

Pixologic. (2020). Zbrush. https://zbrushcore.com/mini/

Saxena, A. (2018). Everything You Need to Know About In-Vehicle Infotainment Systems. https:/www.einfochips.com/blog/everything-you-need-to-know-about-in-vehicle-infotainment-system/

West, D. M. (2016). Avance tecnológico: riesgos y desafios. https://www.bbvaopenmind.com/articulos/avancetecnologico-riesgos-y-desafios/ 